\title{
Desarrollo humano de estudiantes universitarios. Explorando la relación entre usos y aprovechamiento de las TIC y la autonomía personal*
}

\author{
Human Development in College Students. Exploring the Relationship between Uses and Exploitation of the ICTs \\ and the Personal Autonomy \\ Desenvolvimento humano de estudantes universitários. Explorando o relacionamento entre usos e aproveitamento \\ das TIC e a autonomia pessoal
}

\author{
Ana Isabel Zermeño Flores ${ }^{\text {a }}$ \\ Universidad de Colima, México \\ anaz@ucol.mx \\ ORCID: http://orcid.org/0000-0001-7371-8767 \\ Mabel Andrea Navarrete Vega \\ Universidad de Colima, México \\ ORCID: http://orcid.org/0000-0002-5290-3127 \\ Renato González Sánchez \\ Universidad de Colima, México \\ ORCID: http://orcid.org/0000-0003-0737-2838
}

DOI: https://doi.org/10.11144/Javeriana.syp38-74.dheu

Redalyc: http://www.redalyc.org/articulo.oa?id=86059657007

\author{
Fecha de recepción: 29 Mayo 2018 \\ Fecha de aprobación: 30 Noviembre 2018 \\ Fecha de publicación: 18 Junio 2019
}

\section{Resumen:}

Este artículo explora, desde la perspectiva de las tecnologías de información y comunicación para el desarrollo, los usos, aprovechamiento y opiniones sobre las TIC, con el propósito de conocer su incidencia en la autonomía personal de estudiantes universitarios en comunicación. Es un estudio exploratorio, diacrónico y cuantitativo en el que se aplica una encuesta a estudiantes de la Universidad de Colima y la Universidad Autónoma de Coahuila en los años 2015, 2016 y 2017. Entre los principales hallazgos está que ni el acceso al repertorio tecnológico ni las habilidades digitales constituyen condiciones suficientes expandir la autonomía personal en estos jóvenes, como condición del desarrollo humano.

Palabras clave: usos de TIC, desarrollo humano, autonomía personal, jóvenes universitarios, ICT4D.

\section{Abstract:}

This article explores, from the ICT-for-Development perspective, the uses, exploitation and opinions about the ICTs in order to gain insights on how it influences the personal autonomy in college communication students. This is an exploratory, diachronic and quantitative study in which students from the colleges Universidad de Colima and Universidad Autónoma de Cohauila were applied a survey in 2015, 2016 and 2017. One of the main findings is that neither the access to the technological repertoire or the digital skills, are conditions enough to enhance the personal autonomy in this young students, which is a condition for the human development.

Keywords: ICT use, human development, personal autonomy, college youth, ICT4D.

\section{Resumo:}

Este artigo explora, desde a perspectiva das tecnologias da informação e comunicação para o desenvolvimento, os usos, aproveitamento e opiniões sobre as TIC, com o objetivo de conhecer sua incidência na autonomia pessoal de estudantes universitários em comunicação. Trata-se de estudo exploratório, diacrônico e quantitativo no que é aplicado inquérito a estudantes da Universidade de Colima e a Universidade Autónoma de Coahuila em 2015, 2016 e 2017. Entre os principais achados estão que nem o acesso ao repertório tecnológico, nem as habilidades digitais constituem condições suficientes para ampliar a autonomia pessoal dos jovens, como condição do desenvolvimento humano.

Palavras-chave: usos de TIC, desenvolvimento humano, autonomia pessoal, jovens universitários, ICT4D.

Notas de autor:

$$
\text { a Autora de correspondencia. Correo electrónico: anaz@ucol.mx }
$$




\section{Introducción}

Desde la emergencia de las Tecnologías de Información y Comunicación (TIC) -especialmente internety su expansión en las diferentes dimensiones de la vida social, los estudios sobre quién las usa, cómo y para qué, van en incremento. Conocer este universo resulta fundamental por su vínculo con el desarrollo social y humano. Desde la perspectiva del desarrollo a nivel internacional, se entiende que estas tecnologías son relevantes para alcanzar los Objetivos de Desarrollo Sostenible al potenciar la ciencia, la tecnología y la innovación, promover el desarrollo sostenible, intensificar la circulación del conocimiento para la toma de decisiones que mejoren la vida de las personas, así como impulsar el ejercicio de los derechos humanos. En concordancia, la Cumbre Mundial de la Sociedad de la Información en su Declaración de Principios, resalta el papel de los jóvenes como actores estratégicos de desarrollo a los que se debe preparar para enfrentar los desafíos globales del milenio.

Reconocemos que los jóvenes constituyen la fuerza de trabajo del futuro, son los principales creadores de las TIC y también los primeros que las adoptan. En consecuencia, deben fomentarse sus capacidades como estudiantes, desarrolladores, contribuyentes, empresarios y encargados de la adopción de toma de decisiones. (Cumbre Mundial sobre la Sociedad de la Información, 2004)

Desde una perspectiva más reciente, esta mirada converge con la Declaración de Salamanca, que reconoce la incidencia de la revolución tecnológica en las universidades, lo cual "obliga a repensar la función de la universidad y su papel como motor del cambio social y económico” (Poy Solano, 2018). Desde esta óptica, los jóvenes son los actores naturales de la sociedad de la información y el conocimiento (SIyC), por lo que comprender qué sucede con ellos ante estos desafíos resulta decisivo. Sin embargo, independientemente de cómo se comprenda al joven -sospechoso, alternativo o disidente (Reguillo, 2000), como sujeto inmaduro en la antesala de volverse adulto o como actor de desarrollo humano, entre otras opciones (Donas, 2001)-, lo cierto es que en el marco de la vida, tanto en su dimensión biológica como social, y a lo largo de la historia de nuestra especie, los jóvenes han constituido el recurso humano que reemplaza a los viejos. Como especie con visión al futuro, entiende que los jóvenes encarnan la esperanza de mantener la herencia biológica y cultural, pero las expectativas traen consigo miedos, frustraciones o sobrevaloraciones. No es extraño que alrededor de los jóvenes se generen diversos -y a veces contradictorios- significados, expectativas y mitos, algunos de los cuales están en los dominios del desarrollo, lo educativo y lo tecnológico.

Las encuestas sobre adopción de las TIC en el mundo, especialmente sobre internet, reportan al grupo de jóvenes como usuarios sobresalientes por sobre otros segmentos de población (Jiang, 2018), sin embargo, las brechas digitales en sus diferentes dimensiones aún están presentes. A inicios del presente siglo surgieron metáforas como Digital Natives, Digital Nomads, Knowmad, Net Generation que asumieron que los jóvenes, solo por pertenecer a una generación nacida en ecosistemas progresivamente digitalizados, desarrollaban en automático habilidades digitales ad hoc a la SIyC. Estas perspectivas han sido cuestionadas por varios autores que reclaman la necesidad de más estudios empíricos (Brown y Czerniewicz, 2010; Davies, Halford y Gibbins, 2012; Hargittai, 2010; Margaryan, Littlejohn y Vojt, 2011; Zermeño, Alonzo, Ameneyro y Navarrete, 2016).

Según las cifras de adopción de internet, es evidente que la brecha de acceso va en declive, no obstante, se han sumado brechas de segundo y tercer orden (habilidades de uso y aprovechamiento, respectivamente) que vuelve relevante preguntarse: cuando la persona tiene acceso a las TIC, ¿qué hace con ellas?, ¿para qué las usa?, ¿su uso favorece oportunidades para la calidad de vida? y ¿qué hace falta para lograr el cambio?

Respecto a los significados en el ámbito educativo, relevantes para este estudio, en un análisis sobre la vulnerabilidad social de los jóvenes en América Latina, Rodríguez (2001) explica que el sistema educativo, sobre todo el público, pasa por una crisis de legitimidad, porque ya no es garante de la movilidad social y pierde, cada vez más, su papel integrador y nivelador entre las clases sociales. Además de los aspectos que este autor identifica, vemos el fortalecimiento de la oferta de educación no formal y la popularidad de sujetos que 
sin haber terminado la educación superior son exitosos ("Steve Jobs, Mark Zuckerberg y Bill Gates...", 2015. No obstante, la educación como "sistema especializado y dotado de cierta autonomía (... [es] la instancia autorizada para el ejercicio de la función señalada (... respecto de la cual resulta difícil e inconveniente sustraerse" (Rodríguez, 2001, p. 41. Es decir, el sistema educativo sigue siendo la herramienta social a través de la cual las sociedades modernas forman a las nuevas generaciones, las integran y promueven el bienestar. Además, según los jóvenes en México, la educación sigue siendo una alternativa para mejorar su condición de vida. Esto concuerda con la valoración de los jóvenes sobre la educación ( 52,2 $\%$ y el trabajo (25,8 \% como condiciones de éxito en la vida (Instituto Mexicano de la Juventud [IMJUVE], 2012.

Sin soslayar la relevancia de los jóvenes disidentes, alternativos y los excluidos como actores de cambio, para este estudio, interesa observar a los jóvenes universitarios porque se les asocia con ciertos privilegios: son los jóvenes que la sociedad prepara para responder a los desafíos de una sociedad global, digitalizada y con sensibles desigualdades; ser parte del sistema de educación superior amplía el acceso estratégico a conocimiento, redes de contactos, tecnología, etc. Resulta significativo observar a estos jóvenes porque se espera que en un futuro cercano sean los movilizadores de la vida productiva en la sociedad y que aprovechen las TIC para allegarse de conocimiento que genere desarrollo sostenible (Organización de las Naciones Unidas para la Educación, la Ciencia y la Cultura [Unesco], 2015. Sin embargo, en México, aunque el grupo de jóvenes de 15 a 29 años sigue siendo el que tiene mayor presencia 30.600 .000 correspondientes al 25,7 \% respecto de la población total del país (Instituto Nacional de Estadística y Geografía [INEGI], 2015, solo el 10 \% estudia a nivel superior; y resultados "del primer trimestre de 2018 de la ENOE [Encuesta Nacional de Ocupación y Empleo] señalan que de los 15 millones de personas jóvenes ocupadas de 15 a 29 años, el 59,5\% (poco más de 8,9 millones labora en el sector informal" (INEGI, 2018b, p. 4. Estas cifras revelan un panorama nada alentador para el desarrollo de los jóvenes en general. La trampa a la que estos jóvenes se enfrentan es que invirtieron dinero, tiempo, esfuerzo y esperanzas en sus estudios y al egresar -cada vez más-quedan endeudados y sin trabajo, lo que compromete sus metas a futuro (Rossi, 2014.

Según la literatura revisada, los estudios sobre las formas en que los jóvenes usan y apropian las TIC se ha dado preferentemente en el campo educativo porque de entrada se asume que los jóvenes están incorporados al sistema escolar dada su etapa de desarrollo, y que las TIC son relevantes para el aprendizaje. Algunos estudios han mantenido interés en la comprensión de las brechas digitales dado que, como ya se mencionó, las exclusiones se mantienen aun en estos jóvenes que se supone son nativos digitales (Czerniewicz y Brown, 2012; Thinyane, 2010. Los hallazgos develan que el know-how digital no está distribuido al azar entre la población, sino que está relacionado con desigualdades sociales que pueden rastrearse en las familias de origen, así como con el tipo de usos que los jóvenes hacen de esta tecnología (Hargittai, 2010 y la familiaridad, experiencia o tiempo de uso (Brown y Czerniewicz, 2010. De cara al desarrollo humano, la limitante es que estas investigaciones concentran sus preguntas en el ámbito educativo, es decir, en lo que las TIC pueden hacer para mejorar el rendimiento de los jóvenes (Humanante, Conde y García Peñalvo, 2014; Ololube, Agbor, Major, Agabi y Wali, 2016 su socialización (Margaryan et al., 2011 o su gestión y participación en la institución (Ames, 2013; Crovi y López, 2011; Kelling, Kelling y Lennon, 2013.

Sin embargo, para los propósitos de este estudio, al menos se detectan tres problemas asociados al constreñimiento del estudio de los jóvenes y las TIC al ámbito educativo: por un lado, se reduce a los jóvenes, en tanto sujetos de estudio, a su rol de estudiantes, con lo cual se omite la riqueza de su participación en diferentes esferas de pertenencia (familia, amigos, clubes, colectivos, etc., comunidades imaginadas y demás intereses. Por el otro, se reduce a las TIC a herramientas pedagógicas, excluyendo su aportación en la participación social, laboral, cultural, de ocio, entre otras. Otro reduccionismo implícito es que se asume el desarrollo como una aspiración, como una tarea y recompensa futura donde el presente es el tiempo de abonar con el esfuerzo y el joven se prepara para el momento cuando deba participar.

En consecuencia, se detecta la necesidad de abordar este tipo de estudios desde una perspectiva integral: comprendiendo al joven universitario como actor situado en su momento histórico, conectado al espíritu de 
su tiempo (Ortiz Palacios, 1999), en una era informacional (Bell, 2001; Castells, 2000), con participación fuera de la institución universitaria, ampliada a los diferentes ámbitos de la vida. Interesa cuestionarse sobre ¿qué incide en las diferencias de uso y aprovechamiento de las TIC entre los jóvenes, de cara a producir cambios dirigidos a lograr una vida mejor? Lo hasta aquí enunciado, subraya la importancia de comprender la relación jóvenes-TIC-desarrollo, un área que en México poco se ha trabajado y a la que este estudio se propone enriquecer.

\section{Marco teórico}

Este estudio coincide con la línea ICT4D, por sus siglas en inglés (Heeks, 2009), desde la cual se entiende que las TIC son un activo para el desarrollo humano porque: a) son tecnologías intelectuales que generan cambios cognitivos (Bell, 2001), b) permiten acceder y ejercer derechos humanos (Bustamante, 2001) y c) las innovaciones elevan de manera directa la capacidad humana, son una fuente de crecimiento económico al generar productividad y por lo mismo, son un medio para lograr el desarrollo humano (Programa de las Naciones Unidas para el Desarrollo [PNUD], 2001). Paradójicamente, son capaces de ampliar la brecha de las desigualdades cuando no se tiene acceso a ellas (primera brecha digital), cuando no se sabe cómo usarlas (segunda brecha) o cuando no se aprovechan para cambiar situaciones de vida desventajosas (tercera brecha). La investigación sobre la brecha de tercer nivel busca entender quién se beneficia de las formas de uso de internet con respecto a una amplia gama de resultados en la vida offline. Los estudios en TIC4D han de preguntarse cómo los diferentes grupos sociales acceden a las TIC y cómo este acceso contribuye a brindar ventajas en el mundo offline (Cheng, 2013).

Para entender lo que las TIC pueden hacer por el bienestar de las personas, a continuación se sitúa conceptualmente su desarrollo. En los años 80 del siglo XX, la mirada sobre el desarrollo se ajustó y el enfoque del crecimiento económico se desplazó por el de capacidades y justicia social, así se ubicó el horizonte del desarrollo en la riqueza de la vida humana y no en la económica (Bedoya, 2010). En este nuevo paradigma, fueron clave los conceptos de libertades, capacidades y agencia, propuestos por Sen (2010), con lo cual, se concibe el desarrollo humano como proceso que amplía.

La formación de capacidades humanas - tales como un mejor estado de salud, conocimientos y destrezas- y el uso que la gente hace de las capacidades adquiridas para el descanso, la producción, o las actividades sociales, culturales y políticas [...]. Según este concepto de desarrollo humano, el ingreso es sólo (sic) una de las oportunidades que la gente desearía tener, aunque ciertamente muy importante. Pero la vida no solo se reduce a eso. Por lo tanto, el desarrollo debe abarcar más que la expansión de la riqueza y los ingresos. Su objetivo central debe ser el ser humano. (PNUD, 1990, p. 34)

Para entender el nuevo paradigma sobre desarrollo, al que se adscribe este estudio, importan las siguientes consideraciones:

a) Las oportunidades y los satisfactores de las necesidades son ajustables en el tiempo porque cada sociedad valora en diferente forma lo que requiere para lograr el bienestar.

b) No obstante, las capacidades o necesidades para alcanzar una vida digna, son universales (no son relativas a la cultura). Siguiendo a Doyal y Gough (1994), son universales porque refieren a necesidades básicas como la salud o supervivencia (capacidad física y mental para actuar) y la autonomía (capacidad para deliberar, tener conciencia, estar informado, elegir y asumir la responsabilidad del actuar).

c) Para entender el carácter universalista de las necesidades básicas, hay que distinguirlas de los deseos individuales o apetencias "que son objetivos derivados de las preferencias particulares del individuo y del entorno cultural” (Gough, 2007, p. 187). 
d) La autonomía para la acción no es individual, su satisfacción se da en un entorno social que construye el habitus (Davies et al., 2012), el cual permite desarrollar y descubrir el propio potencial, y se vincula a las formas en las que imaginamos cómo aprovechar las TIC.

e) Este entorno social está atravesado por cuatro precondiciones sociales (producción, reproducción, transmisión cultural y autoridad política), que cualquier grupo social que pretende sobrevivir y prosperar en el tiempo debe atender (Braybrooke, 1987, citado por Doyal y Gough, 1994).

Cuando Doyal y Gough (1994) afirman que "ser autónomos [...] es tener la habilidad para hacer elecciones y tomar decisiones informadas sobre lo que debería hacerse y cómo hacerlo” (p. 186), resulta ineludible ver a las TIC como uno de los satisfactores potenciales de esta necesidad. Por ello, se insiste en la importancia de conocer las elecciones que los estudiantes hacen sobre cuáles TIC usan y cómo las aprovechan (sea en la escuela, el trabajo, la vida civil, etc.).

Los usos de las TIC implican no solo lo lúdico, esparcimiento o sociabilidad, sino el uso productivo (relacionado con la primera precondición social de Braybrooke, 1987). Para este estudio, sin demérito de los otros usos, importa el uso productivo como aquel que expande la autonomía personal en los jóvenes para favorecer su acción humana y su participación en la vida social, con el fin de desarrollar su potencial personal y colectivo. Por lo tanto, importa observar acciones concretas que suponen elecciones personales, como el uso del tiempo libre, el uso de las TIC, así como las opiniones sobre tales tecnologías.

La autonomía, al ser una condición previa de la acción e interacción humanas, implica la participación en la vida social, que no se reduce a lo político o al activismo, sino que refiere a ser parte activa de la dinámica social en la cual las personas encuentran o no, oportunidades y condiciones necesarias para desarrollar su potencial.

\section{Marco metodológico}

El estudio es exploratorio, diacrónico y cuantitativo. Se aplicó una encuesta a estudiantes de pregrado de dos universidades mexicanas: Universidad de Colima y Universidad Autónoma de Coahuila (campus Torreón); en ambos casos, a estudiantes de Comunicación al inicio de sus primeros semestres. Se escogió esta etapa de formación para identificar cuál es su cultura digital al inicio de su carrera y su relación con la vida offline. En una fase posterior del proyecto, se hará una siguiente medición, cuando estos jóvenes estén en el último semestre de sus estudios para observar su evolución desde una perspectiva comparativa.

La aplicación en línea al universo de estudiantes (163) de primer ingreso de Comunicación se llevó a cabo en tres momentos: en 2015, a 44 estudiantes; en 2016, a 35 y en 2017, a 84. El cuestionario se diseñó con preguntas de opción múltiple, con escala de Likert y abiertas. Se examinaron datos demográficos de ambos progenitores/tutores, demográficos de los jóvenes, repertorio tecnológico (accesos, dispositivos y aplicaciones -apps-), usos de las TIC, participación en la vida social y opiniones sobre el papel de las TIC en lo escolar, profesional, sociedad actual, así como sobre internet y redes sociales.

\section{Métodos estadísticos empleados}

Además del análisis descriptivo de frecuencias (tabla 1) y el univariable, se empleó el análisis de correspondencias (AC) simple y exploratorio. El AC "es una técnica factorial (...) [que resume] una gran cantidad de datos en un número reducido de dimensiones, con la menor pérdida de información” (AbadGonzález, Muñiz-Martínez y Cervantes-Blanco, 2008, p. 363). La técnica permite observar relaciones de cercanía entre características o individuos agrupados en diversas variables. Estas últimas pueden ser ordinales o incluso nominales, favoreciendo flexibilidad a la técnica, pues se prescinde de determinar la distribución de probabilidad de las variables involucradas. Como lo menciona Peña (2002, p. 193), es "equivalente al 
análisis de componentes principales para variables cualitativas". En investigaciones que recogen información cualitativa de los encuestados, se hace necesaria la reparametrización ${ }^{1}$ de las variables.

TABLA 1

Frecuencias de las variables involucradas en el estudio

\begin{tabular}{|c|c|c|c|c|c|c|c|}
\hline Variable & Items & F & $\%$ & Variables & Ítems & $\mathbf{F}$ & $\%$ \\
\hline \multirow[t]{2}{*}{ Sexo } & Mujer & 99 & 58,6 & \multirow[t]{2}{*}{ Estado civil } & Casado & 2 & 1,2 \\
\hline & Hombre & 64 & 37,9 & & Soltero & 161 & 95,3 \\
\hline \multirow[t]{3}{*}{ Ańo de aplicación } & 2015 & 44 & 26 & \multirow{3}{*}{$\begin{array}{l}\text { Sistema } \\
\text { operativo } \\
\text { preferente }\end{array}$} & Android & 105 & 62,1 \\
\hline & 2016 & 35 & 20,7 & & IOS & 41 & 24,3 \\
\hline & 2017 & 84 & 49,7 & & Diversos u otros & 17 & 10,1 \\
\hline \multirow[t]{5}{*}{$\begin{array}{l}\text { Ocupación del } \\
\text { padre }\end{array}$} & Ama de casa & 3 & 1,8 & \multirow[t]{5}{*}{$\begin{array}{l}\text { Educación del } \\
\text { padre }\end{array}$} & $\begin{array}{l}\text { Sin educ. formal / } \\
\text { Primaria }\end{array}$ & 31 & 18,3 \\
\hline & No calificada & 20 & 11,8 & & Secundaria & 27 & 16 \\
\hline & Operativa & 54 & 32 & & Preparatoria & 42 & 24,9 \\
\hline & Técnica & 42 & 24,9 & & Licenciatura & 47 & 27,8 \\
\hline & $\begin{array}{l}\text { Cientifico- } \\
\text { profesional }\end{array}$ & 44 & 26 & & Posgrado & 16 & 9,5 \\
\hline \multirow[t]{5}{*}{$\begin{array}{l}\text { Ocupación de la } \\
\text { madre }\end{array}$} & Ama de casa & 64 & 37,9 & \multirow[t]{5}{*}{$\begin{array}{l}\text { Educación de } \\
\text { la madre }\end{array}$} & $\begin{array}{l}\text { Sin educ. formal / } \\
\text { Primaria }\end{array}$ & 22 & 13 \\
\hline & No calificada & 18 & 10,7 & & Secundaria & 40 & 23,7 \\
\hline & Operativa & 28 & 16,6 & & Preparatoria & 43 & 25,4 \\
\hline & Técnica & 28 & 16,6 & & Licenciatura & 47 & 27,8 \\
\hline & $\begin{array}{l}\text { Cientifico- } \\
\text { profesional }\end{array}$ & 25 & 14,8 & & Posgrado & 11 & 6,5 \\
\hline \multirow[t]{4}{*}{$\begin{array}{l}\text { Además de } \\
\text { estudiar, ¿qué otra } \\
\text { ocupación realizas? }\end{array}$} & Solo estudiar & 64 & 37,9 & \multirow{4}{*}{$\begin{array}{l}\text { ¿Qué } \\
\text { actividades de } \\
\text { ocio sueles } \\
\text { realizar? }\end{array}$} & $\begin{array}{l}\text { Actividades } \\
\text { preponderantes de } \\
\text { ocio }\end{array}$ & 118 & 69,8 \\
\hline & Ayudar en casa & 9 & 5,3 & & $\begin{array}{l}\text { Prácticas de vida } \\
\text { social o de puertas } \\
\text { afuera }\end{array}$ & 12 & 7,1 \\
\hline & Trabajar & 50 & 29,6 & & $\begin{array}{l}\text { Actividades } \\
\text { deportivas }\end{array}$ & 20 & 11,8 \\
\hline & $\begin{array}{l}\text { Politica, arte, } \\
\text { deporte, servicio } \\
\text { comunitario }\end{array}$ & 40 & 23,7 & & $\begin{array}{l}\text { Potencial } \\
\text { intelectual } \\
\text { productivo-crítico y } \\
\text { creativo }\end{array}$ & 13 & 7,7 \\
\hline \multirow{4}{*}{$\begin{array}{l}\text { Participa en } \\
\text { movimientos } \\
\text { sociales }\end{array}$} & Nunca & 81 & 47,9 & \multirow{4}{*}{$\begin{array}{l}\text { Usa redes } \\
\text { sociales para } \\
\text { promover } \\
\text { activismo } \\
\text { social }\end{array}$} & Nunca & 60 & 35,5 \\
\hline & Rara vez & 51 & 30,2 & & Rara vez & 66 & 39,1 \\
\hline & Cierta frecuencia & 22 & 13 & & Cierta frecuencia & 25 & 14,8 \\
\hline & Frecuentemen-te & 9 & 5,3 & & Frecuentemente & 12 & 7,1 \\
\hline
\end{tabular}


TABLA 1 (CONT.)

Frecuencias de las variables involucradas en el estudio

\begin{tabular}{|c|c|c|c|c|c|c|c|}
\hline Variable & Ítems & $\mathbf{F}$ & $\%$ & Variables & Ítems & F & $\%$ \\
\hline \multirow{4}{*}{$\begin{array}{l}\text { Realiza } \\
\text { contenidos } \\
\text { digitales }\end{array}$} & Nunca & 20 & 11,8 & \multirow{4}{*}{$\begin{array}{l}\text { ¿Cuál es la } \\
\text { razón por la } \\
\text { que realizas } \\
\text { contenido? }\end{array}$} & No ha hecho & 18 & 10,7 \\
\hline & Rara vez & 47 & 27,8 & & $\begin{array}{l}\text { Ocio } \\
\text { entretenimiento }\end{array}$ & 42 & 24,9 \\
\hline & Cierta frecuencia & 68 & 40,2 & & $\begin{array}{l}\text { Tarea o trabajo } \\
\text { escolar }\end{array}$ & 79 & 46,7 \\
\hline & Frecuentemen-te & 28 & 16,6 & & $\begin{array}{l}\text { Empleo, } \\
\text { conocimiento, } \\
\text { apoyo social }\end{array}$ & 24 & 14,2 \\
\hline \multirow{5}{*}{$\begin{array}{l}\text { Opinión del papel } \\
\text { de las TIC en la } \\
\text { educación }\end{array}$} & Relevantes & 77 & 47,2 & \multirow{5}{*}{$\begin{array}{l}\text { Opinión del } \\
\text { papel de las } \\
\text { TIC en la vida } \\
\text { profesional }\end{array}$} & Relevantes & 60 & 36,8 \\
\hline & Eficientes & 11 & 6,7 & & Eficientes & 20 & 12,3 \\
\hline & Riesgo potencial & 19 & 11,7 & & $\begin{array}{l}\text { Pertinentes con } \\
\text { riesgo potencial }\end{array}$ & 11 & 6,7 \\
\hline & Útiles & 54 & 33,1 & & Útiles & 69 & 42,3 \\
\hline & Otra & 2 & 1,2 & & Otro & 3 & 1,8 \\
\hline \multirow{5}{*}{$\begin{array}{l}\text { Opinión del papel } \\
\text { de las TIC en la } \\
\text { sociedad actual }\end{array}$} & Relevantes & 51 & 31,3 & \multirow{5}{*}{$\begin{array}{l}\text { Opinión sobre } \\
\text { el internet }\end{array}$} & Relevantes & 55 & 33,7 \\
\hline & Eficientes & 3 & 1,8 & & Eficientes & 3 & 1,8 \\
\hline & $\begin{array}{l}\text { Pertinentes con } \\
\text { riesgo potencial }\end{array}$ & 59 & 36,2 & & $\begin{array}{l}\text { Pertinentes con } \\
\text { riesgo potencial }\end{array}$ & 50 & 30,7 \\
\hline & Útiles & 41 & 25,2 & & Útiles & 52 & 31,9 \\
\hline & Otra & 9 & 5,5 & & Otros & 3 & 1,8 \\
\hline \multirow{5}{*}{$\begin{array}{l}\text { Opinión sobre las } \\
\text { redes sociales }\end{array}$} & Relevantes & 17 & 10,4 & & & & \\
\hline & Eficientes & 2 & 1,2 & & & & \\
\hline & $\begin{array}{l}\text { Pertinentes con } \\
\text { riesgo potencial }\end{array}$ & 84 & 51,5 & & & & \\
\hline & Útriles & 56 & 34,4 & & & & \\
\hline & Otra & 4 & 2,5 & & & & \\
\hline
\end{tabular}

Fuente: elaboración propia a partir de datos de 163 encuestas

El AC se apoya en diferentes estadísticos de prueba, pero en este trabajo se emplean solo dos: la inercia, que se equipara a la varianza o información retenida por los ejes vectoriales resultantes y una prueba de ji cuadrada. Esta última tiene como hipótesis nula $\left(\mathrm{H}_{0}\right)$ la ausencia de asociación entre las variables involucradas; si la significancia asintótica para un valor de ji cuadrada (con determinado número de grados de libertad) es menor que 0,05 , se rechaza dicha hipótesis nula al $95 \%$ de confianza. Este estadístico permite presentar en el trabajo solo a aquellas relaciones significativas de las diversas variables involucradas en el estudio. Finalmente, se empleó la correlación Tau de Kendall y/o la de Spearman, para conocer el comportamiento simétrico de variables ordinales involucradas en el AC. Estos estadísticos estiman la correlación entre variables ordinales, de tal manera que un valor positivo indica que ambas variables aumentan o disminuyen conjuntamente, mientras que un valor negativo indica que al incrementar una variable, la otra disminuye.

Asimismo, se empleó el análisis de componentes principales (APC) exploratorios, el cual reduce el número de variables originales que presentan una alta correlación (que podrían parecer redundantes), en un conjunto menor de variables intrínsecas con menor pérdida de varianza o información. En el presente estudio, las variables originales corresponden al número de aplicaciones de diferentes tipos y propósitos. La finalidad es incorporar los resultados del APC al modelo de regresión como variables explicativas.

Finalmente, se emplea un modelo de regresión logit ordenado, el cual tiene como variable dependiente la frecuencia de creación de los contenidos digitales (opciones: 1: nunca, 2: rara vez crea, 3: crea con cierta frecuencia y 4: crea frecuentemente). Este es un modelo de probabilidad, por lo cual se consideró necesario estimar las elasticidades o efectos marginales para que se exprese la probabilidad de que los alumnos estén 
en alguna de las cuatro categorías descritas. Algunas características socioeconómicas de los estudiantes o sus preferencias de actividades extraescolares y sociales fueron las variables independientes o explicativas.

\section{Resultados}

Los datos permiten identificar una paradoja en la adopción de las TIC por parte de los estudiantes. Estos jóvenes tienen un acceso amplio a las TIC, como lo muestra su repertorio tecnológico (tabla 2), han desarrollado habilidades digitales visibles en prácticas de creación de contenidos (tabla 1) ${ }^{2}$, pero aun así no presentan usos productivos relevantes que repercutan favorablemente en la ampliación de su autonomía personal (como se verá más adelante).

TABLA 2

Repertorio tecnológico y prácticas asociadas de los jóvenes (2015, 2016 y 2017)

\begin{tabular}{|c|c|c|c|c|c|c|c|c|}
\hline & & $\begin{array}{r}\text { Mujeres } \\
(99) \\
\end{array}$ & $\begin{array}{r}\text { Hombres } \\
(64) \\
\end{array}$ & $M$ & $\begin{array}{r}2015 \\
(44) \\
\end{array}$ & $\begin{array}{r}2016 \\
(35) \\
\end{array}$ & $\begin{array}{r}2017 \\
(84) \\
\end{array}$ & $M$ \\
\hline \multirow[t]{4}{*}{ Dispositivos } & $\begin{array}{l}\text { Computadora o } \\
\text { laptop }\end{array}$ & 88,9 & 85,9 & 87,4 & 93,2 & 85,7 & 85,7 & 88,2 \\
\hline & Tableta & 32,3 & 32,8 & 32,6 & 36,4 & 20 & 35,7 & 30,7 \\
\hline & Celular & 99 & 98,4 & 98,7 & 100 & 97,1 & 98,8 & 98,7 \\
\hline & $\begin{array}{l}\text { Consola de } \\
\text { videojuego }\end{array}$ & 20,2 & 39,1 & 29,6 & 22,7 & 17,1 & 34,5 & 24,8 \\
\hline \multirow[t]{6}{*}{$\begin{array}{l}\text { Acceso a } \\
\text { internet }\end{array}$} & $\begin{array}{l}\text { Cualquier lugar } \\
\text { mediante el } \\
\text { celular }\end{array}$ & 70,7 & 85,9 & 78,3 & 59 & 69 & 89 & 72,3 \\
\hline & $\begin{array}{l}\text { Cualquier lugar } \\
\text { mediante } \\
\text { dispositivos } \\
\text { móviles }\end{array}$ & 11,1 & 28,1 & 19,6 & 14 & 16 & 17 & 19 \\
\hline & Mi casa & 92,9 & 87,5 & 90,2 & 98 & 86 & 89 & 91 \\
\hline & El trabajo & 12,1 & 18,8 & 15,4 & 23 & 0 & 17 & 13,3 \\
\hline & La escuela & 80,8 & 70,3 & 75,6 & 91 & 63 & 63 & 81,7 \\
\hline & $\begin{array}{l}\text { Acceso } \\
\text { comunitario }\end{array}$ & 21,2 & 9,4 & 15,3 & 55 & 3 & 2 & 20 \\
\hline
\end{tabular}

Fuente: elaboración propia a partir de datos de 163 encuestas

Nota: Datos en porcentajes.

Este resultado coincide con el estudio realizado por Berrío-Zapata y Rojas-Hernández (2014), quienes encuentran que "los estudiantes [universitarios] tienen acceso a las condiciones instrumentales asociadas con la utilización de las TIC pero no desarrollan su utilidad productiva" (p. 138). Subrayamos: esta disposición no es solo hacia la expertise tecnológica sino al aprovechamiento de su autonomía personal.

Los ecosistemas digitales de los estudiantes son cada vez más ubicuos gracias a la expansión del uso del celular (Czerniewicz y Brown, 2012). En el 2015, solo el $59 \%$ de los estudiantes accedía a internet a través del celular; para el 2017, aumentó 30 puntos (llegó a $89 \%$ ), esto ha significado reducir la preferencia de acceso de otros lugares como el hogar y la escuela. Esta tendencia concuerda con los resultados de Puspitasari e Ishii (2016), quienes identifican un salto de etapas móviles, es decir, un proceso mediante el cual los nuevos usuarios de internet acceden a través del celular y no a través del PC. También encuentra eco en las encuestas de INEGI (2017) que señalan al celular como "la tecnología de mayor penetración nacional y con las menores diferencias regionales" (p. 6); además, del aumento de acceso a internet mediante el celular (92\% de los que tienen celular), según INEGI (2018a, p. 2). 
La creación de contenidos digitales por los estudiantes es una práctica que puede indicar la forma como construyen y ejercen su autonomía personal, en la medida en que eligen qué producir y para qué hacerlo. Esta práctica presenta variaciones que se asocian o explican tanto por las razones de su creación como por la opinión que tengan sobre las TIC en la vida profesional, así como por las actividades extraescolares que realizan (figura 1. Los estudiantes que frecuentemente producen contenidos digitales son aquellos que tienen ocupaciones asociadas a la política, el arte, los deportes y el servicio comunitario, mientras que los que lo realizan con cierta frecuencia son aquellos que, además de estudiar, trabajan. Por otra parte, los que consideran a las TIC como útiles en la vida profesional crean contenidos con cierta frecuencia; sin embargo, de manera paradójica, aquellos que nunca han creado contenidos consideran las TIC como eficientes.
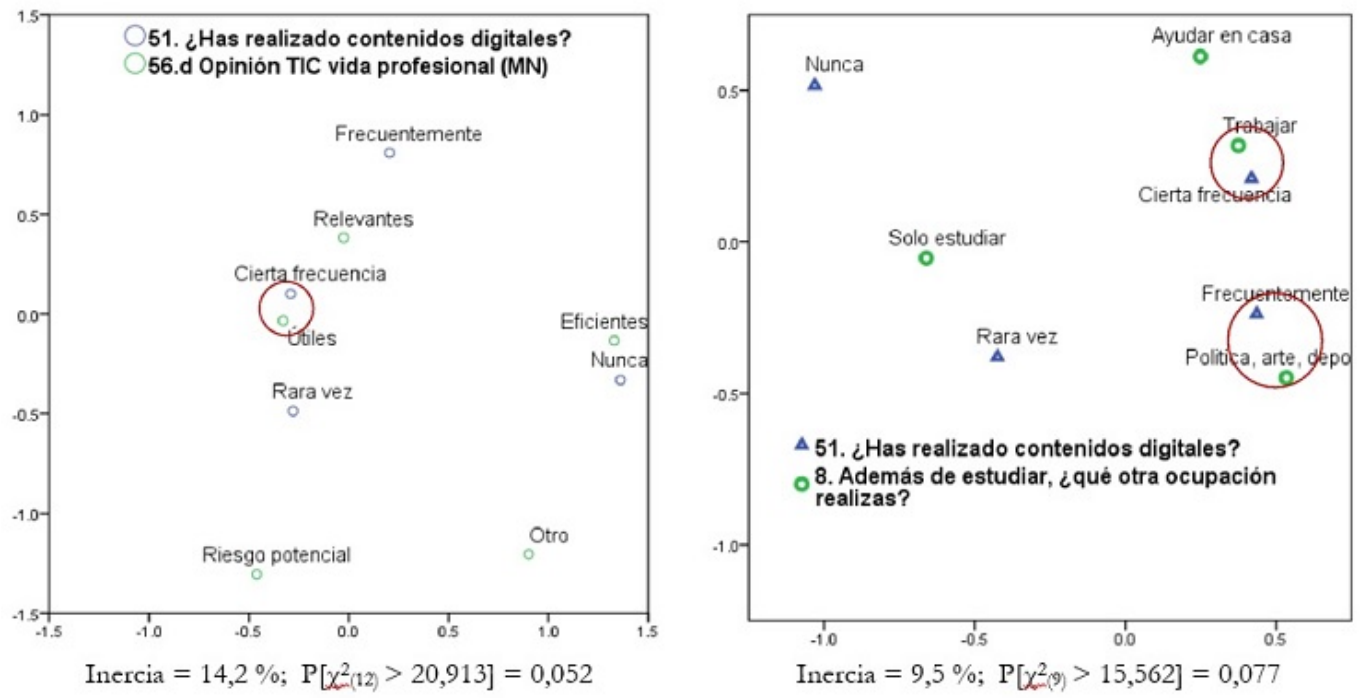

FIGURA 1

Razón de crear contenidos digitales con otras variables

Fuente: elaboración propia

En la figura 2, se relacionan algunas variables de involucramiento en movimientos sociales de estudiantes y TIC. Destaca una polarización en la participación en movimientos sociales y el uso de redes sociales por parte de los estudiantes para promocionar estas actividades. A pesar del alto uso de redes sociales, este no se vincula a los movimientos sociales. Aquellos que asiduamente participan son quienes también con frecuencia emplean las redes sociales con fines de promoción de su activismo social; mientras que aquellos que afirmaron que nunca se involucran en estos movimientos son también los que jamás promueven estas actividades (o muy raramente). Los estadísticos de simetría indican que al aumentar el involucramiento social aumenta la promoción. Este resultado puede parecer obvio, no obstante, es relevante hacer notar la redundancia entre lo offline y lo online, lo cual evidencia la necesidad del compromiso y participación social offline que luego puede, o no, reflejarse en el activismo online. Estos resultados concuerdan fuertemente con los obtenidos por Padilla (2014), quien encuentra una baja participación política online por parte de estudiantes universitarios en Aguascalientes. Cabe notar que la participación social offline reiterada, solo se observa en un número bajo de estudiantes. Esto confirma que los usos de las TIC por los estudiantes no están incidiendo en su participación social -y viceversa-. 

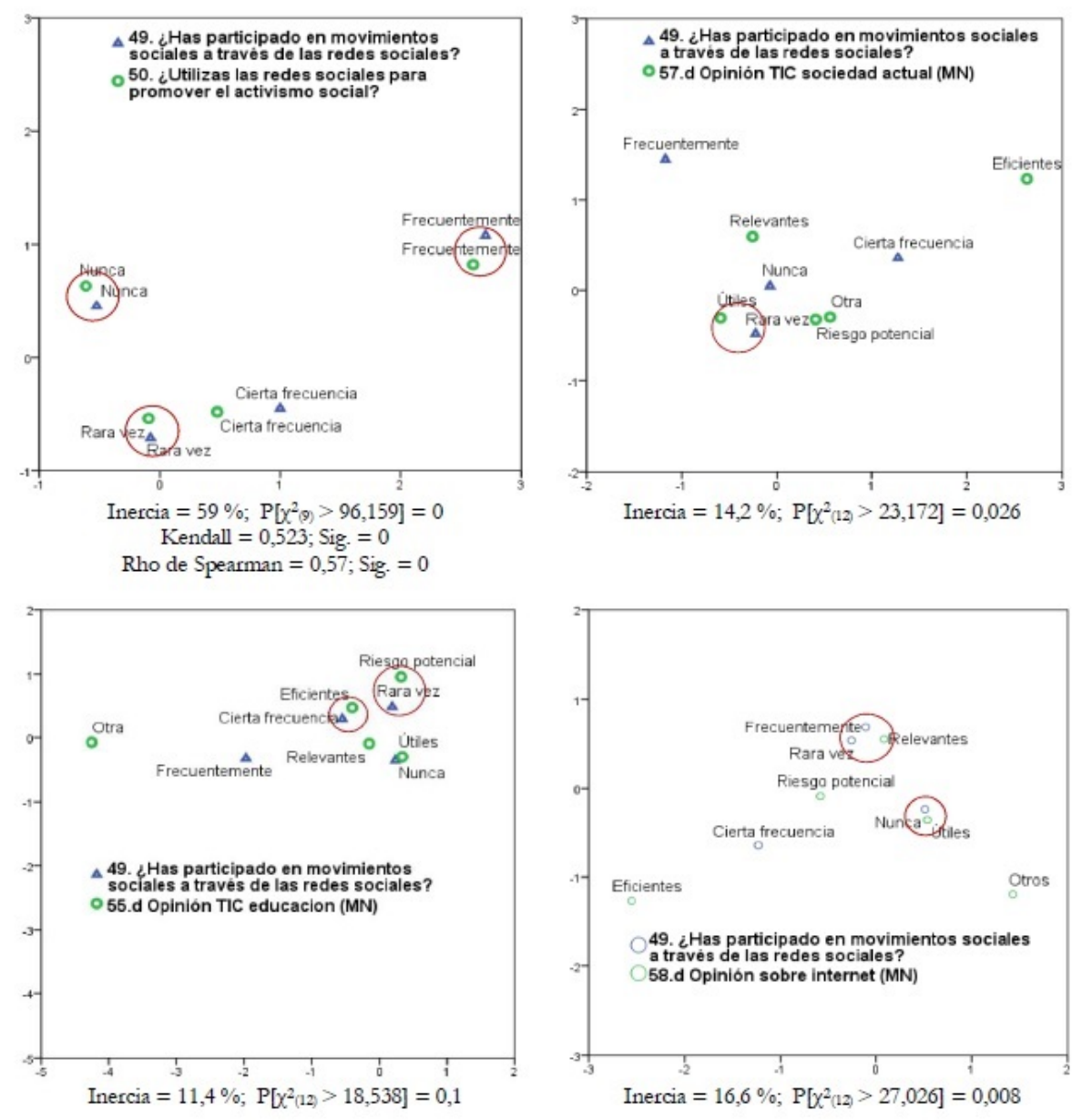

FIGURA 2

La participación social de estudiante y las TIC

Fuente: elaboración propia

El resto de los paneles de la figura 2 muestran la asociación de cercanía entre la participación en movimientos sociales del estudiante y sus opiniones sobre las TIC. Aquellos que raramente participan consideran a las TIC en la sociedad como útiles; los que rara vez participan consideran al papel de las TIC como pertinentes en la educación, pero con algún riesgo potencial; mientras que los que participan con cierta frecuencia las consideran como eficientes. Por último, los estudiantes que consideran al papel del internet como relevante, rara o frecuentemente se involucran en los movimientos sociales.

Sin embargo, los estudiantes en general consideran que internet es relevante para la sociedad, lo que concuerda con el último estudio del Pew Research Center (Jiang, 2018). No obstante, la opinión sobre las TIC relacionada con el tipo de actividades u ocupaciones de los jóvenes, presenta matices. En la Figura 3, se muestra la relación de cercanía entre las actividades fuera de la escuela y las opiniones del estudiante sobre las TIC. Si el encuestado solo estudia, considera al papel de las TIC en la sociedad (o en la vida profesional) como útil, mientras que si trabaja lo aprecia como relevante. Finalmente, si desarrolla actividades asociadas a la política, el arte, el deporte o el servicio comunitario, juzga el rol de las TIC en la sociedad como pertinentes, pero con algún riesgo potencial. Esto nos coloca en una caja negra que requiere replantear las preguntas y el análisis en futuros estudios. 

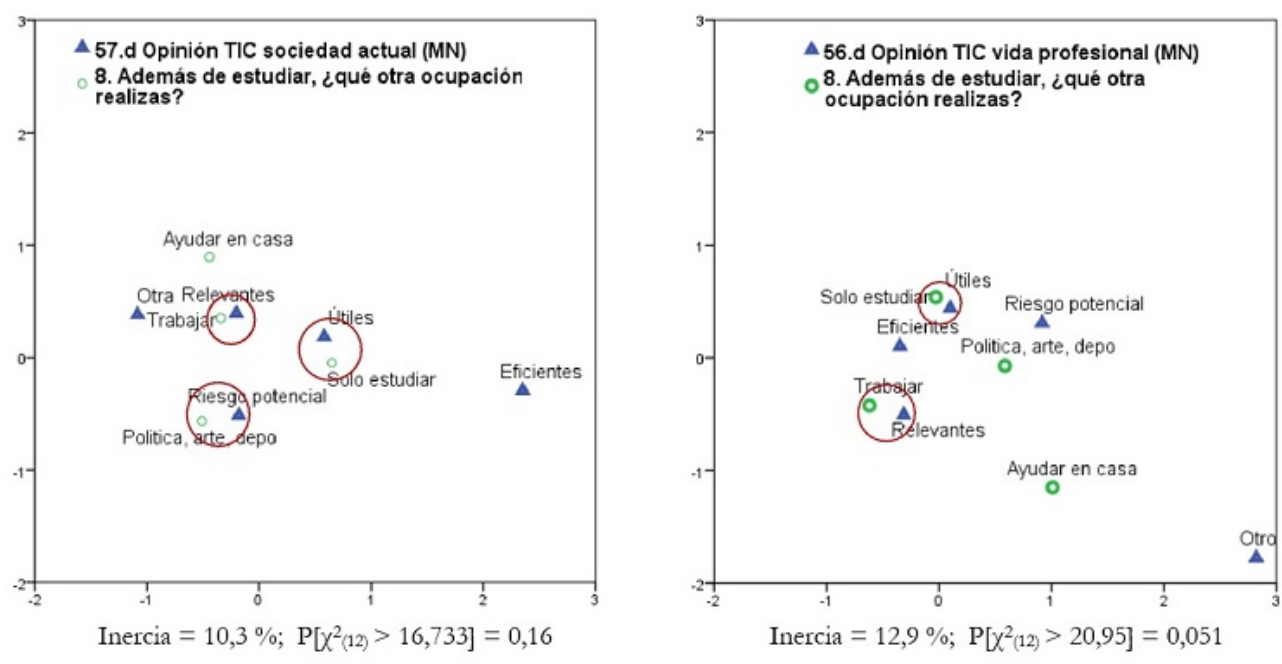

FIGURA 3

La ocupación extraescolar del estudiante y sus opiniones sobre las TIC Fuente: elaboración propia

\section{Análisis de componentes principales exploratorios}

A partir de nueve variables originales altamente correlacionadas, el ACP generó dos variables intrínsecas, reteniendo el $62 \%$ de la varianza. Solo una variable fue descartada de las estimaciones, por aportar poca información o varianza al modelo (número de aplicaciones primordiales en el teléfono celular). Las nuevas variables intrínsecas se renombraron a partir de las variables originales que agrupan (tabla 3). Como se observa, la agrupación se basa en valores de cargas factoriales mayores a 5,5. Por otra parte, las variables intrínsecas presentan un valor de alfa de Cronbach cercano a 0,8 , lo cual permite confiar en ellas para posteriores análisis estadísticos, que en este caso es para análisis de regresión. Los nombres asignados a las variables fueron:

- $\quad \mathrm{CPR} 1=$ Aplicaciones (apps) totales de entretenimiento, sociales e instaladas hace tres meses.

- $\quad \mathrm{CPR} 2=$ Apps tipo utilitarias, productivas, educativas, informáticas, de servicios y de creación.

TABLA 3

Resultados de análisis de componentes principales

\begin{tabular}{lrrr}
\hline \multicolumn{1}{c}{ Variables originales } & CPR1 & CPR2 & Comunalidades \\
\hline $\begin{array}{l}\text { Número total de aplicaciones }(a p p s) \text { que tienes instaladas en tu } \\
\text { celular }\end{array}$ & 0,621 & & 0,678 \\
\hline Número de apps de entretenimiento & 0,577 & & 0,498 \\
\hline Número de apps sociales & 0,623 & & 0,532 \\
\hline Número de apps utilitarias y de productividad & & 0,826 & 0,739 \\
\hline Número de apps educativas e informativas & & 0,856 & 0,739 \\
\hline Número de apps de servicios & 0,615 & 0,453 \\
\hline Número de apps para creación & & 0,568 & 0,61 \\
\hline Número de apps primordiales en tu celular & & & \\
\hline Número de apps que has instalado en los últimos 3 meses & 0,836 & & 0,701 \\
\hline Proporción de la varianza & 0,279 & 0,34 & \\
\hline Varianza acumulada & 0,279 & 0,619 & \\
\hline Alfa de Cronbach (confiabilidad) & 0,791 & 0,821 & \\
\hline
\end{tabular}




\section{Modelo logit ordenado}

Se eligió este modelo debido a que la variable dependiente presenta una tendencia creciente en la frecuencia de creación de contenidos digitales; además, variables como el comportamiento de los estudiantes en el uso de TIC (número de dispositivos, aplicaciones, horas en internet, razones de creación, etc.) o en sus actividades extraescolares o de ocio, así como características socioeconómicas (edad, educación y ocupación de padres, etc.) pueden influir en la frecuencia de creación de contenidos digitales. Las pruebas estadísticas del modelo completo permiten afirmar que es posible hacer predicciones con este; asimismo, los interceptos resultaron significativos, lo que implica que la manera como se distribuyen las opciones de la variable dependiente es adecuada.

Como se observa (tabla 4), el número de actividades que el estudiante realiza en internet influye en la creación de contenidos; así, por cada nueva actividad, se incrementa en 0,2 \% la probabilidad de que cree frecuentemente contenido o en $1,5 \%$ de que produzca contenido con cierta frecuencia. De manera similar, el que cuente con un mayor número de aplicaciones utilitarias (productivas, educativas, etc.) aumenta la probabilidad de que el alumno realice contenido con cierta frecuencia o frecuentemente. La variable que más influye en la creación de contenidos es la razón por la que los estudiantes los realizan. Así, por ejemplo, si tienen la finalidad de crearlos por ocio o entretenimiento, aumenta la probabilidad en $20,2 \%$ de que lo hagan de manera frecuente; si el motivo es por tareas o trabajos escolares, incrementa la probabilidad en 14,7 $\%$ de que los realicen de manera frecuente y, si el motivo de creación es por su actividad laboral, obtener conocimiento o apoyo social, la probabilidad se acrecienta en 10,1 \% en esta opción.

La ocupación del padre también influye en las frecuencias de creación de contenido. Si el padre se dedica a actividades científico-profesionales, aumenta en 17,5\% la probabilidad de que el estudiante realice con cierta frecuencia contenido digital; en cambio, si el padre se dedica a actividades operativas, esta probabilidad alcanza el 10,2 \%. De acuerdo con este modelo, variables como la ocupación de la madre, las ocupaciones laborales de los estudiantes o bien el tipo de actividades de ocio que realizan los jóvenes no influyen en su frecuencia en la creación de contenido digital; de igual manera, el número de dispositivos, las horas que pasa en internet al día, el sexo o la edad tampoco lo hacen. 
TABLA 4

Modelos de regresión logit ordinal para frecuencia de creación de contenido digital

\begin{tabular}{|c|c|c|c|c|c|c|c|c|c|c|c|c|c|c|c|}
\hline \multirow[b]{2}{*}{ Variable } & \multicolumn{4}{|c|}{ Coeficientes } & \multicolumn{2}{|c|}{ Nunca crea } & \multicolumn{3}{|c|}{ Rara vez crea } & \multicolumn{3}{|c|}{$\begin{array}{r}\text { Crea con cierta } \\
\text { frecuencia }\end{array}$} & \multicolumn{3}{|c|}{ Crea frecuentement } \\
\hline & Coef. & EE & Sig. & Elast. & EE & Sig. & Elast. & EE & Sig. & Elast. & EE & Sig. & Elast. & EE & Sig. \\
\hline Número dispositivos & $-0,083$ & 0,216 & & 0,006 & 0,016 & & 0,006 & 0,015 & & $-0,01$ & 0,027 & & $-0,001$ & 0,003 & \\
\hline $\begin{array}{l}\text { Número de actividades } \\
\text { en internet }\end{array}$ & 0,118 & 0,045 & *ook & $-0,009$ & 0,005 & * & $-0,008$ & 0,005 & & 0,015 & 0,006 & $* *$ & 0,002 & 0,001 & $* *$ \\
\hline $\begin{array}{l}\text { Promedio de horas al día } \\
\text { en que usa internet }\end{array}$ & $-0,035$ & 0,042 & & 0,003 & 0,003 & & 0,002 & 0,003 & & $-0,004$ & 0,005 & & $-0,001$ & 0,001 & \\
\hline $\begin{array}{l}\text { FR1: Apps totales o de } \\
\text { entretenimiento, sociales } \\
\text { e instaladas hace } 3 \text { meses }\end{array}$ & $-0,195$ & 0,272 & & 0,014 & 0,021 & & 0,013 & 0,02 & & $-0,025$ & 0,034 & & $-0,003$ & 0,004 & \\
\hline $\begin{array}{l}\text { FR2: Apps tipo } \\
\text { utilitarias, productivas, } \\
\text { educativas, informáticas, } \\
\text { de servicios y de } \\
\text { creación }\end{array}$ & 0,397 & 0,194 & ** & $-0,029$ & 0,02 & & $-0,027$ & 0,02 & & 0,05 & 0,025 & $* *$ & 0,006 & 0,003 & $* *$ \\
\hline Sexo masculino, si $=1$ & 0,234 & 0,384 & & $-0,017$ & 0,029 & & $-0,016$ & 0,029 & & 0,029 & 0,049 & & 0,004 & 0,006 & \\
\hline Edad del estudiante & 0,13 & 0,117 & & $-0,009$ & 0,009 & & $-0,009$ & 0,01 & & 0,016 & 0,015 & & 0,002 & 0,002 & \\
\hline $\begin{array}{l}\operatorname{RCC}(1) \text { : ocio o } \\
\text { entretenimiento, si }=1\end{array}$ & 22,009 & 0,957 & $* * *$ & $-0,994$ & 0,004 & $* * * *$ & 0,233 & 0,067 & $* * * x$ & 0,559 & 0,049 & **** & 0,202 & 0,062 & $* * * *$ \\
\hline $\begin{array}{l}\mathrm{RCC}(1) \text { : tareas o trabajos } \\
\text { escolares, si }=1\end{array}$ & 21,622 & 0,919 & $* * *$ & $-0,992$ & 0,006 & $* * *$ & 0,308 & 0,056 & $* * *$ & 0,537 & 0,051 & **** & 0,147 & 0,038 & $* * *$ \\
\hline $\begin{array}{l}\mathrm{RCC}(1) \text { : empleo, } \\
\text { conocimiento o apoyo } \\
\text { social, si = } 1\end{array}$ & 21,192 & 1,009 & 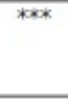 & $-0,988$ & 0,01 & *ack & 0,403 & 0,114 & *akx & 0,484 & 0,082 & xikik & 0,101 & 0,048 & kik* \\
\hline Variable & Coef. & EE & Sig. & Elast. & EE & Sig. & Elast. & EE & Sig. & Elast. & EE & Sig. & Elast. & EE & Sig. \\
\hline $\mathrm{OP}(2)$ : operativa, si $=1$ & 0,997 & 0,567 & * & $-0,096$ & 0,067 & & $-0,018$ & 0,054 & & 0,102 & 0,056 & * & 0,012 & 0,006 & $*$ \\
\hline $\mathrm{OP}(2):$ técnica, si $=1$ & 0,364 & 0,554 & & $-0,044$ & 0,07 & & 0,011 & 0,028 & & 0,029 & 0,043 & & 0,003 & 0,005 & \\
\hline $\begin{array}{l}\mathrm{OP}(2) \text { : científico- } \\
\text { profesional, si }=1\end{array}$ & 1,458 & 0,656 & $* *$ & $-0,12$ & 0,076 & & $-0,078$ & 0,075 & & 0,175 & 0,082 & $* *$ & 0,023 & 0,013 & $*$ \\
\hline $\mathrm{OM}(3)$ : operativa, si $=1$ & 0,582 & 0,531 & & $-0,038$ & 0,038 & & $-0,052$ & 0,06 & & 0,079 & 0,078 & & 0,011 & 0,011 & \\
\hline $\mathrm{OM}(3):$ técnica, si $=1$ & $-0,36$ & 0,525 & & 0,035 & 0,054 & & 0,006 & 0,018 & & $-0,036$ & 0,05 & & $-0,004$ & 0,006 & \\
\hline $\begin{array}{l}\text { OM(3): científico- } \\
\text { profesional, si = } 1\end{array}$ & 0,76 & 0,603 & & $-0,046$ & 0,04 & & $-0,078$ & 0,082 & & 0,109 & 0,095 & & 0,015 & 0,014 & \\
\hline $\begin{array}{l}\operatorname{AEE}(4) \text { : ayuda en casa, } \\
\text { si }=1\end{array}$ & $-0,935$ & 0,823 & & 0,122 & 0,146 & & $-0,045$ & 0,104 & & $-0,07$ & 0,05 & & $-0,007$ & 0,005 & \\
\hline $\operatorname{AEE}(4):$ trabaja, si $=1$ & 0,696 & 0,466 & & $-0,048$ & 0,042 & & $-0,055$ & 0,044 & & 0,091 & 0,063 & & 0,012 & 0,009 & \\
\hline $\begin{array}{l}\text { AEE(4): incursiona en } \\
\text { política, arte, deporte, } \\
\text { acts. comunitarias, si }=1\end{array}$ & 0,48 & 0,509 & & $-0,036$ & 0,043 & & $-0,03$ & 0,039 & & 0,059 & 0,065 & & 0,007 & 0,009 & \\
\hline $\begin{array}{l}\text { AOE(5): vida social de } \\
\text { puertas afuera }\end{array}$ & 0,042 & 0,787 & & $-0,003$ & 0,052 & & $-0,004$ & 0,068 & & 0,006 & 0,106 & & 0,001 & 0,014 & \\
\hline $\begin{array}{l}\text { AOE }(5) \text { : actividades } \\
\text { deportivas }\end{array}$ & $-0,37$ & 0,51 & & 0,03 & 0,045 & & 0,019 & 0,025 & & $-0,044$ & 0,056 & & $-0,005$ & 0,007 & \\
\hline $\begin{array}{l}\mathrm{AOE}(5) \text { : potencial } \\
\text { intelectual productivo, } \\
\text { crítico y creativo }\end{array}$ & $-0,451$ & 0,758 & & 0,037 & 0,074 & & 0,021 & 0,025 & & $-0,052$ & 0,079 & & $-0,006$ & 0,009 & \\
\hline Intercepto 1 & 21,258 & 2,437 & $* * *$ & & & & & & & & & & & & \\
\hline Intercepto 2 & 25,292 & 2,537 & $* * *$ & & & & & & & & & & & & \\
\hline Intercepto 3 & 27,824 & 2,618 & $* * *$ & & & & & & & & & & & & \\
\hline
\end{tabular}

Fuente: elaboración propia a partir de datos de 163 encuestas

Notas: $\mathrm{CC}(1)=$ Razón por la que crea contenidos; $\mathrm{OP}(2)=$ Tipo de ocupación del padre; $\mathrm{OM}(3)=$ Tipo de ocupación de la madre; AEE(4) = Además de estudiar el alumno se ocupa; AOE(5) = Actividades de ocio del estudiante. Estadísticas del modelo completo: Log pseudolikelihood = -140,17617; Wald chi $^{2}(22)=973,8$; Prob $>$ chi $^{2}=0$; Pseudo R2 $=0,3299$. Los asteriscos representan significancia al: $\left({ }^{*}\right) 90 \%,\left({ }^{* *}\right) 95 \%$ y $\left({ }^{* *}\right) 99 \%$. 


\section{Conclusiones}

La exploración sobre las formas en las que los estudiantes de comunicación aprovechan sus ecosistemas, habilidades y conocimientos digitales, así como su entorno familiar, les ayuda y potencia su capacidad creativa para la producción mediática digital; sin embargo, esta producción está vinculada mayoritariamente a aspectos lúdicos y de sociabilidad y menos a la participación de la vida en sociedad, como condición de autonomía personal. Darle seguimiento a las prácticas digitales resulta útil para investigaciones en este campo, porque indica en qué se invierte el tiempo y esfuerzo cuando se usan las TIC, preferentemente internet. Estas decisiones revelan la capacidad de elección de las personas como indicador de la conformación de su autonomía personal. En el caso de los jóvenes estudiados, las elecciones sobre las TIC no parecen expandir su autonomía en aspectos relacionados con la participación en la vida social, ni en la capacidad para distinguir oportunidades y elegir las mejores sobre opciones informadas. No obstante la aportación de estos resultados, se requieren estudios de mayor extensión y profundidad para caracterizar mejor la autonomía personal de los universitarios, y sobre ese tejido, identificar la incidencia de las TIC, entre otros satisfactores.

El epicentro del ecosistema digital es el teléfono celular, dada su rápida adopción y las posibilidades que brinda, sobre todo cuando se conecta a internet, ya que permite mayor portabilidad, menor costo, conectividad constante, ubicuidad y uso personal, lo que resulta en ventajas muy atractivas sobre para estos jóvenes que disfrutan de actividades sociales.

Si bien falta un seguimiento de mayor tiempo y profundidad en la relación de jóvenes y las TIC, diferenciada por género de los padres, se concluye que hay indicios de una brecha que no está relacionada con el acceso, sino con las estructuras mentales, con el habitus (Davies et al., 2012), y que se hace evidente en las formas de adoptar y aprovecharlas. Los imaginarios sobre lo que se puede lograr con estas, se relaciona con una racionalidad tecnológica que orienta juicios, valores, formas de ser y estar en el mundo, por lo tanto, implica lo que los estudiantes imaginan que pueden alcanzar y transformar con esas herramientas. De manera particular, se visualiza la necesidad de profundizar sobre el peso de la familia, en específico de los progenitores, en las disposiciones de los jóvenes para la creación de contenidos digitales y en los imaginarios de sus alcances con las TIC. Es posible que el peso de la madre sea mayor en otras prácticas sociales de los universitarios, pero en este estudio, los resultados muestran que no tienen incidencia; habrá que buscar las razones, replantear las preguntas y los análisis.

Respecto al estudio de la relación entre TIC y desarrollo humano, este trabajo permite vislumbrar que se requiere desenfocar en "lo tecnológico" (como catalizador de cambios por sí mismo) para colocar en el primer plano las prácticas cotidianas y el habitus como actores de la vida social y generadores de sentido. La aproximación requiere un planteamiento básico sobre ¿qué incide en las diferencias de uso y aprovechamiento de las TIC entre las personas, de cara a producir cambios dirigidos a lograr una vida mejor?

Los resultados refuerzan la recomendación de atender la variable de TIC, cuando se estudia a los jóvenes, subrayando que esto resulta más propicio cuando se trata de los universitarios, ya que estos jóvenes tienen cada vez más mayor acceso a las TIC, así como capacidades digitales. Las TIC representan un ámbito poderoso donde se expresan, socializan y se construyen. Los retos están en cómo inciden en el desarrollo de su potencial.

Finalmente, parece que la relación entre el uso de las TIC y el fortalecimiento de la autonomía personal de los universitarios analizados encuentra eco en aquellos que tienen una vida offline enriquecida. Esto sugiere la necesidad de que los programas de Gobierno, educativos o de la sociedad civil, dedicados a promover el desarrollo integral de los comunicólogos, desarrollen estrategias eficientes y de amplio espectro 
que contemplen prácticas intelectuales, creativas, de participación ciudadana y justicia social, lo cual puede abonar en la formación de mejores profesionales y ciudadanos.

\section{Referencias}

Abad-González, J., Muñiz-Martínez, N., y Cervantes-Blanco, M. (2008. Análisis de correspondencias simples y múltiples. En J. P. Lévy-Mangin, J. Varela-Mallou y J. Abad-Gonza\#lez (coords.), Análisis multivariable para la ciencias sociales (pp. 361-416). Madrid: Pearson Educación.

Ames, M. (2013, febrero). Managing mobile multitasking: The culture of iPhones on Stanford campus. Trabajo presentado en el Computer Supported Cooperative Work 2013, San Antonio, Texas. Recuperado de http:// www.morganya.org/research/ames-cscw13-iphone.pdf

Bedoya, C. L. (2010). Amartya Sen y el desarrollo humano. Memorias, 8(13), 277-288.

Bell, D. (2001). El advenimiento de la sociedad post-industrial: un intento de prognosis social. Madrid: Alianza.

Berrío-Zapata, C., y Rojas-Hernández, H. (2014). The digital divide in the university: The appropriation of ICT in higher education students from Bogota, Colombia. Comunicar, 22(43), 133-142. https://doi.org/10.3916/C4 3-2014-13

Brown, C., y Czerniewicz, L. (2010). Debunking the digital native: Beyond digital apartheid, towards digital democracy. Journal of Computer Assisted Learning, 26(5), 357-369. https://doi.org/10.1111/j.1365-2729.201 0.00369. $\mathrm{x}$

Bustamante, J. (2001). Hacia la cuarta generacio\#n de derechos humanos: repensando la condicio\#n humana en la sociedad tecnolo\#gica [Edición especial: La Sociedad de la Información]. Revista Iberoamericana de Ciencia, Tecnologia, Sociedad e Innovación, 1, 3-4. Recuperado de http://www.oei.es/revistactsi/numero1/bustamante. htm

Castells, M. (2000). La era de la información: economía, sociedad y cultura (Elpoder dela identidad). México: Siglo XXI.

Cheng, W. (2013). The implications of social capital for the digital divides in America. The Information Society, 29, 13-25. https://doi.org/10.1080/01972243.2012.739265

Crovi, D., y López, R. (2011). Tejiendo voces: jóvenes universitarios opinan sobre la apropiación de internet en la vida académica. Revista Mexicana de Ciencias Politicas y Sociales, 11(212), 69-80. https://doi.org/10.22201/fcpys.2 448492xe.2011.212.30421

Cumbre Mundial sobre la Sociedad de la Información. (2004). Declaración de principios. Construir la sociedad de la información: un desafio global para el nuevo milenio (Documento WSIS-03/GENEVA/4-S). Ginebra: Unión Internacional de Telecomunicaciones. Recuperado de http://www.itu.int/net/wsis/docs/geneva/official/dop-e s.html

Czerniewicz, L., y Brown, C. (2012). The habitus of digital "strangers" in higher education. British Journal of Educational Technology, 44(1), 44-53. https://doi.org/10.1111/j.1467-8535.2012.01281.x

Davies, H., Halford, S., y Gibbins, N. (2012, junio). Digital Natives? Investigating young people's critical skills in evaluating web based information. Trabajo presentado en la Web Science 2012, WebSci '12, Evanston, Illinois.

Donas, S. (ed.). (2001). Adolescencia y juventud en América Latina. Cartago: Libro Universitario Regional.

Doyal, L., y Gough, I. (1994). La teoria de las necesidades. Barcelona: Icaria.

Gough, I. (2007). El enfoque de las capacidades de M. Nussbaum: un análisis comparado con nuestra teoría de las necesidades humanas. Papeles de Relaciones Ecosociales y Cambio Global, 100, 177-202. Recuperado de https:// dialnet.unirioja.es/servlet/articulo?codigo $=2515036$

Hargittai, E. (2010). Digital $\mathrm{Na}(\mathrm{t})$ ives? Variation in internet skills and uses among members of the "Net Generation" (L. Nagore y S. Silva, Trads.). Sociological Inquiry, 80(1), 92-113. https://doi.org/10.1111/j.1475-682X.2009.003 17.x

Heeks, R. (2009). The ICT4D 2.0 Manifesto: Where next for ICTs and international development? (Documento de trabajo N.o 42). Manchester, UK: Development Informatics Group/Institute for Development Policy and 
Management. Recuperado de http://www.seed.manchester.ac.uk/subjects/idpm/research/publications/wp/di /di-wp42/

Humanante, P. R., Conde, M. A., y García Peñalvo, F. J. (2014, junio). Differences and similarities in use and acceptance of PLEs between universities in Ecuador and Spain. En H. M. Fardoun y J. A. Gallud (eds.), IDEE'14 Proceedings of the 2014 Workshop on Interaction Design in Educational Environments (pp. 1-70). Nueva York: ACM Publications. https://doi.org/10.1145/2643604.2643609

Instituto Mexicano de la Juventud. (2012). Encuesta Nacional de Valores en Juventud 2012. Resultados generales. México: Autor. Recuperado de https://www.imjuventud.gob.mx/imgs/uploads/ENVAJ_2012.pdf

Instituto Nacional de Estadística y Geografía (INEGI). (2015). Encuesta Intercensal 2015. México: Autor. Recuperado de http://www.beta.inegi.org.mx/proyectos/enchogares/especiales/intercensal/

Instituto Nacional de Estadística y Geografía (INEGI). (2017). Estadísticas a propósito del... día mundial de internet (17 de mayo). Datos nacionales. Aguascalientes, MX: Autor. Recuperado de http://www.diputados.gob.mx/sed ia/biblio/usieg/comunicados/educ_cien_tec-b.pdf

Instituto Nacional de Estadística y Geografía (INEGI). (2018a). En México 71.3 millones de usuarios de internet y 17.4 millones de hogares con conexión a este servicio: Encuesta Nacional sobre Disponibilidad y Uso de Tecnologias de la Información en los Hogares 2017 (Comunicado de prensa N.o 105/18). Aguascalientes, MX: Autor. Recuperado de http://www.beta.inegi.org.mx/contenidos/saladeprensa/boletines/2018/OtrTemEcon/ ENDUTIH2018_02.pdf

Instituto Nacional de Estadística y Geografía (INEGI). (2018b). Estadísticas a propósito del... día internacional de la juventud (12 de agosto). Datos nacionales (Comunicado de prensa N.o 350/18). Aguascalientes, MX: Autor. Recuperado de https://www.inegi.org.mx/contenidos/saladeprensa/aproposito/2018/juventud2018_Nal.pdf

Jiang, J. (2018, mayo). Millennials stand out for their technology use, but older generations also embrace digital life. Washington, DC: Pew Research Center. Recuperado de http://www.pewresearch.org/fact-tank/2018/05/02/ millennials-stand-out-for-their-technology-use-but-older-generations-also-embrace-digital-life/

Kelling, N., Kelling, A., y Lennon, J. (2013). The tweets that killed a university: A case study investigating the use of traditional and social media in the closure of a state university. Computers in Human Behavior, 29(6), 2656-2664. https://doi.org/10.1016/j.chb.2013.06.044

Margaryan, A., Littlejohn, A., y Vojt, G. (2011). Are digital natives a myth or reality? University students' use of digital technologies. Computers \& Education, 56, 429-440. https://doi.org/10.1016/j.compedu.2010.09.004

Ololube, N., Agbor, C., Major, N., Agabi, C., y Wali, W. (2016). 2015 Global Information Technology Report: Consequences on knowledge management in higher education institutions in Nigeria. International Journal of Education and Development using Information and Communication Technology, 12(2), 4-25. Recuperado de ht tps://files.eric.ed.gov/fulltext/EJ1111464.pdf

Organización de las Naciones Unidas para la Educación, la Ciencia y la Cultura (Unesco). (2015). Objetivos de Desarrollo Sostenible para la Comunicación y la Información [A/RES/70/125. Documento final de la reunión de alto nivel de la Asamblea General de Naciones Unidas sobre el examen general de la aplicación de los resultados de la Cumbre Mundial sobre la Sociedad de la Información, celebrada en Nueva York los días 15 y 16 de diciembre de 2015]. Recuperado de http://es.unesco.org/sdgs/ci

Ortiz Palacios, L. A. (1999). Acción, significado y estructura en la teoría de A. Giddens. Convergencia. Revista de Ciencias Sociales, 20, 57-84. Recuperado de https://convergencia.uaemex.mx/article/view/1864

Padilla, R. (2014). Ciudadanía política en la red. Análisis de las prácticas políticas entre jóvenes universitarios. Comunicación y Sociedad, 21, 71-100. https://doi.org/10.32870/cys.v0i21.572

Peña, D. (2002). Análisis de datos multivariantes. Madrid: McGraw Hill.

Poy Solano, L. (2018, 22 de mayo). Universidades promoverán modelo con rumbo digital. La Jornada.com//Sociedad y Justicia. Recuperado de https://www.jornada.com.mx/ultimas/2018/05/22/universidades-promoveran-mod elo-con-rumbo-digital-1464.html 
Programa de las Naciones Unidas para el Desarrollo (PNUD). (1990). Desarrollo humano. Informe 1990 (Primer informe anual, A. García, Trad.). Bogotá: Tercer Mundo. Recuperado de http://hdr.undp.org/sites/default/file s/hdr_1990_es_completo_nostats.pdf

Programa de las Naciones Unidas para el Desarrollo (PNUD). (2001). Informe sobre desarrollo humano 2001: poner el adelanto tecnológico al servicio del desarrollo humano. México: Ediciones Mundi-Prensa. Recuperado de http: //hdr.undp.org/sites/default/files/hdr_2001_es.pdf

Puspitasari, L., e Ishii, K. (2016). Digital divides and mobile Internet in Indonesia: Impact of smartphones. Telematics and Informatics, 33(2), 472-483. https://doi.org/10.1016/j.tele.2015.11.001

Reguillo, R. (2000). Emergencias de culturas juveniles. Bogotá: Norma.

Rodríguez, J. (2001). Vulnerabilidad y grupos vulnerables: un marco de referencia conceptual mirando a los jo\#venes (Cepal. Serie Población y desarrollo N.o 17). Santiago de Chile: Cepal. Recuperado de https://repositorio.cep al.org/bitstream/handle/11362/7150/S018659_es.pdf

Rossi, A. (productor y director). (2014). Ivory tower [Película]. Park City, Salt Lake City, Utah, US: Paramount Pictures.

Sen, A. K. (2010). Desarrollo y libertad. Buenos Aires: Planeta.

“Steve Jobs, Mark Zuckerberg y Bill Gates, sin título universitario. Revelación de un reciente estudio”. (2015, 16 de abril). Clarin.com/Mundo. Recuperado de https://www.clarin.com/mundo/steve-jobs-zuckerberg-bill-gatessin-titulo-universitario_0_BJ9HijFP7l.html

Thinyane, H. (2010). Are digital natives a world-wide phenomenon? An investigation into South African first year students' use and experience with technology. Computers \& Education, 55(1), 406-414. https://doi.org/10.101 6/j.compedu.2010.02.005

Zermeño, A., Alonzo, R., Ameneyro, A., y Navarrete, M. (2016). La inclusión digital para la inclusión social en jóvenes. En A. Cruz-Manjarrez, M. Chávez y A. Zermeño (eds.), Los jóvenes en el mundo actual: deconstrucción de las nuevas realidades (pp. 343-368). México: Universidad de Colima/UNAM.

\section{Notas}

* Artículo de investigación científica Este artículo deriva del “Observatorio sobre usos productivos de las TIC", proyecto que surge en 2015 y es coordinado desde Agorante, grupo de investigación en sociedad y tecnologías del Centro Universitario de Investigaciones Sociales, de la Universidad de Colima. Agradecimientos: A la Dra. Blanca Chong López, de la Universidad Autónoma de Coahuila, por su apoyo en la aplicación de encuesta.

1 En este trabajo, el término se emplea para indicar la asignación de valores numéricos a las respuestas de los estudiantes encuestados. Así, los autores interpretaron las respuestas asignándoles un número, con lo cual las respuestas se convierten en variables ordinales o nominales. De este modo, se hace más eficiente la aplicación de métodos estadísticos.

2 La tabla 1 describe ciertas características de la muestra de los estudiantes encuestados, útil para la comprensión del proceder metodológico; no obstante, en este apartado de resultados, se brinda información sobre algunas formas de uso que se exploraron en la encuesta.

\section{Licencia Creative Commons CC BY 4.0}

Cómo citar este artículo: Zermeño Flores, A. I., Navarrete Vega, M. A., y González Sánchez, R. (2019). Desarrollo humano de estudiantes universitarios. Explorando la relación entre usos y aprovechamiento de las TIC y la autonomía personal. Signo y Pensamiento, 38(74). https://doi.org/10.11144/Javeriana.syp3874.dheu 